Revue d'histoire de l'Amérique française

REYUE D.HISTOIRE DE L'AMÉRIQUE FRANÇAISE

\title{
Stratification sociale et différenciation spatiale en milieu urbain pré-industriel
} Le cas des locataires montréalais, 1731-1741

\section{Daniel Massicotte}

\section{Volume 44, numéro 1, été 1990}

URI : https://id.erudit.org/iderudit/304863ar

DOI : https://doi.org/10.7202/304863ar

Aller au sommaire du numéro

\section{Éditeur(s)}

Institut d'histoire de l'Amérique française

\section{ISSN}

0035-2357 (imprimé)

1492-1383 (numérique)

Découvrir la revue

\section{Citer cet article}

Massicotte, D. (1990). Stratification sociale et différenciation spatiale en milieu urbain pré-industriel : le cas des locataires montréalais, 1731-1741. Revue d'histoire de l'Amérique française, 44(1), 61-83. https://doi.org/10.7202/304863ar

\section{Résumé de l'article}

L'historiographie américaine des villes pré-industrielles et industrielles a accordé une grande attention aux thèmes de la stratification sociale et de la ségrégation spatiale. Les débats concernant la chronologie ont fait émerger les concepts-clés d'égalitarisme social et d'intégration spatiale appliqués à la ville pré-industrielle. L’objectif du présent article est de vérifier la validité de ces concepts pour le cas montréalais en analysant la composition socio-professionnelle des locataires, leur répartition dans la ville et la cartographie des loyers. Nos résultats de recherche tendent à invalider ces concepts car les locataires montréalais de 1731-1741 montrent une structure socioprofessionnelle découpée en trois groupes hiérarchiques principaux où chacun tend à se concentrer dans des lieux distincts de la ville selon un modèle concentrique. C'est dans le fait que le faible loyer des plus démunis représente une part importante de leur revenu tandis que le loyer coûteux des gens aisés n'en représente qu'une faible part que la division sociale des locataires s'opère véritablement.
Tous droits réservés @ Institut d'histoire de l'Amérique française, 1990
Ce document est protégé par la loi sur le droit d'auteur. L'utilisation des services d'Érudit (y compris la reproduction) est assujettie à sa politique d'utilisation que vous pouvez consulter en ligne.

https://apropos.erudit.org/fr/usagers/politique-dutilisation/ 


\title{
STRATIFICATION SOCIALE ET DIFFÉRENCIATION SPATIALE EN MILIEU URBAIN PRÉ-INDUSTRIEL: LE CAS DES LOCATAIRES MONTRÉALAIS, 1731-1741 ${ }^{1}$
}

\author{
DANIEL MASSICOTTE \\ Département d' histoire \\ Université de Montréal
}

\begin{abstract}
RÉSUMÉ
L'historiographie américaine des villes pré-industrielles et industrielles a accordé une grande attention aux thèmes de la stratification sociale et de la ségrégation spatiale. Les débats concernant la chronologie ont fait émerger les concepts-clés d'égalitarisme social et d'intégration spatiale appliqués à la ville pré-industrielle. L'objectif du présent article est de vérifier la validité de ces concepts pour le cas montréalais en analysant la composition socio-professionnelle des locataires, leur répartition dans la ville et la cartographie des loyers. Nos résultats de recherche tendent à invalider ces concepts car les locataires montréalais de 1731-1741 montrent une structure socioprofessionnelle découpée en trois groupes hiérarchiques principaux où chacun tend à se concentrer dans des lieux distincts de la ville selon un modèle concentrique. C'est dans le fait que le faible loyer des plus démunis représente une part importante de leur revenu tandis que le loyer coûteux des gens aisés n'en représente qu'une faible part que la division sociale des locataires s'opère véritablement.
\end{abstract}

\begin{abstract}
Social stratification and spatial segregation have been important themes in american urban history. For the pre-industrial city the key concepts adopted are those of social egalitarianism and spatial integration. This article seeks to verify the validity of these concepts with regards to Montreal by analysing the socio-professional status of tenants, their distribution in the city and the geographical distribution of rental costs. Our results do not support the application of these concepts. Montreal tenants in the period 1731-1741 were divided into three distinct socio-professional groups each of which congregated in a distinct area according to a concentric pattern. The determinant factor for social segregation seems to have been the amount of rent paid: the poor paid a large part of their income whereas the higher rents of the rich represented a small portion of their revenue.
\end{abstract}

\section{INTRODUCTION}

La société urbaine du Canada pré-industriel constitue un champ d'étude encore peu exploré malgré quelques travaux récents privilé-

1 Ce texte présente quelques conclusions de notre mémoire de maîtrise, Le marché du logement locatif à Montréal de 1731 à 1741 (Département d'histoire, Université de Montréal, 1987). Nous tenons à remercier nos directeurs de recherche, messieurs John A. Dickinson et Pierre Tousignant, de même que messieurs Jean Blain et Roch Legault, pour leurs judicieux commentaires. La préparation de cet article a été rendu possible grầce à l'appui financier de la Société canadienne d'hypothèque et de logement. 
giant les perspectives sociales et économiques qui montrent un intérêt certain pour la compréhension de la vie urbaine. La présente étude s'intéresse plus spécifiquement au problème de la stratification sociale et de sa traduction en un espace différencié à partir d'une analyse de la répartition des locataires et des niveaux de loyers dans le quadrillage urbain. Elle cherche à la fois à intégrer le cas montréalais dans une perspective nord-américaine en recourant aux travaux des historiens américains et à lier la ville pré-industrielle à la ville industrielle dans la longue durée.

\section{1 - BILAN HISTORIOGRAPHIQUE}

L'historiographie traditionnelle canadienne a brossé un tableau plutôt descriptif de la sociabilité urbaine en s'inspirant de l'oeuvre de Charlevoix, du récit de voyage de Pehr Kalm et autres écrits d'observateurs contemporains ${ }^{2}$. Les travaux de ces historiens ne montrent aucun souci de saisir la structure sociale ${ }^{3}$, bien que certains aient identifié quelques métiers et groupes sociaux ${ }^{4}$. Sous leur plume, la ville préindustrielle apparaît comme une société peu différenciée ne comportant pas de système hiérarchique. Quelques-uns ont certes tenté d'identifier la fonction première de quelques rues et secteurs urbains, mais aucun ne s'est penché de façon systématique sur l'organisation spatiale ${ }^{5}$.

En omettant de signaler l'existence de propriétaires et de locataires, l'historiographie traditionnelle a jusqu'à très récemment transmis l'idée préconçue que la ville pré-industrielle est habitée par une masse de petits propriétaires. Il faut dire que l'absence de recensements et d'enquêtes sur l'état des logis montréalais durant cette période deux types de sources particulièrement utilisées pour l'époque industrielle - a découragé la recherche sur ce sujet. Deux articles seulement nous offrent des évaluations de la proportion des locataires à Montréal,

2 P.-F.-X. de Charlevoix, Histoire et description générale de la Nouvelle-France (Paris, Nyon Fils libraire, 1744, Montréal, Éditions Élysées, 1976), tome 3: 138; Voyage de Pehr Kalm au Canada en 1749, traduction annotée du journal de route par Jacques Rousseau et Guy Béthune (Montréal, Pierre Tisseyre, 1977).

3 Voir A. Leblond de Brumath, Histoire populaire de Montréal depuis son origine jusqu'à nos jours (Montréal, Granger Frères, 1890), 196-198, 213, 239, 292; W. H. Atherton, Montreal (1534-1914) (Montréal, Clarke, 1914), 1: 344-345, 359-364; J. Bruchési, De Ville-Marie à Montréal (Montréal, Éd. de l'arbre, 1942), 77-78, 86, 100; G. Lanctôt, Montréal au temps de la Nouvelle-France, 1642-1760 (Montréal, Ducharme, 1942), 22-23.

4 Voir C. Bertrand, Histoire de Montréal. Tome 1: $1535-1760$ (Montréal, Beauchemin, 1935), 57-62, 87-88, 199-201; E. R. Adair, «The Evolution of Montreal Under the French Regime», Canadian Historical Association Annual Report (1942), 24, 34; L. Roberts, Montreal: From Mission Colony to World City (Toronto, Macmillan, 1969), 75; R. Rumilly, Histoire de Montréal (Montréal, Fides, 1970), 1: 89-90, 325, 341-342, 381-382, 403.

5 La récente étude d'André Lachance sur les villes canadiennes se rapproche en quelque sorte de l'historiographie traditionnelle car bien qu'il observe des indices de cloisonnement social, l'auteur insiste davantage sur le système de relations et l'intégration sociale et économique des différents groupes urbains. A. Lachance, La vie urbaine en Nouvelle-France (Montréal, Boréal, 1987), 43-68. 
qui serait de $30 \%$ en 1741 et de $69 \%$ en $1825^{6}$. Mentionnons aussi deux études portant sur la propriété fonçière dans la période pré-industrielle ${ }^{7}$. Les travaux sur les locataires ont surtout porté sur la seconde moitié du XIXe siècle et la première partie du XXe siècle et ils abordent ces problèmes dans le cadre spécifique du procès d'industrialisation ${ }^{8}$. Ainsi le problème général de l'inégalité sociale et le problème particulier du logement locatif semblent naître avec l'industrialisation, alors qu'en réalité ils lui sont bien antérieurs, comme les travaux récents portant sur les commencements de Montréal le laissent entrevoir ${ }^{9}$ et comme nous allons tenter de le démontrer nous-même.

L'historiographie récente sur la ville de Québec a mieux couvert la structure sociale et l'organisation spatiale ${ }^{10}$, grâce en particulier à des sources plus nombreuses, dont une série de recensements, qui permet-

6 L. Dechêne, «La croissance de Montréal au XVIIIe siècle», Revue d' histoire de l'Amérique française, 27,2 (septembre 1973): 169; J.-P Bernard, P.-A. Linteau et J.-C Robert, «Les tablettes statistiques de Jacques Viger (1825)», GRSM, Rapport 1972-73 (Montréal, Université du Québec à Montréal, 1973), 21. R. Sweeny estime, quant à lui, que cette dernière proportion serait probablement trop élevée: «Un passé en mutation: bilan et perspectives pour une histoire socio-économique de Montréal au XIXe siècle», J.-R. Brault, dir.., Montréal au XIXe siècle. Des gens, des idées, des arts, une ville, Actes du colloque organisé par la Société historique de Montréal, automne 1988 (Ottawa, Leméac éditeur, 1990), 27-28.

7 P.-A. Linteau et J.-C. Robert, «Propriété foncière et société à Montréal: une hypothèse», $R H A F, 28,1$ (juin 1974): 17-39; Alan M. Stewart, «Settling in 18th Century Faubourg: Property and Family in the Saint-Laurent Suburb, 1735-1810», mémoire de maîtrise (histoire), Université McGill, 1988.

8 H. B. Ames, The City Below the Hill: a Sociological Study of a Portion of the City of Montreal (Toronto, University of Toronto Press, 1972) [1897]; J.-C. Robert, Montréal, 1821 1871. Aspects de l'urbanisation, thèse de doctorat, École des hautes études en sciences sociales (Paris), 1977, 491 p.; T. Copp, Classe ouvrière et pauvreté: les conditions de vie des travailleurs montréalais, 1897-1929 (Montréal, Boréal Express, 1978), 213 p.; M. Bellavance et J.-D. Gronoff, "Les structures de l'espace montréalais à l'époque de la Confédération", Cahiers de géographie du Québec, 24,63 (décembre 1980): 363-384; M. H. Choko, Crises du logement à Montréal, 1860-1939 (Montréal, Éditions coopératives Albert Saint-Martin, 1980), 282 p.; P.-A. Linteau, Maisonneuve ou Comment des promoteurs fabriquent une ville (Montréal, Boréal Express, 1981), 280 p.; D. Hanna et S. Olson, «Métiers, loyers et bouts de rue: l'armature de la société montréalaise de 1881 à 1901», Cahiers de géographie du Québec, 27,71 (septembre 1983): 255275; B. Bradbury, The Working Class Family Economy, Montreal 1861-1881, thèse de doctorat, Université Concordia, 1984), 554 p.; D. B. Hanna, «The Layered City: a Revolution in Housing in Mid-Nineteenth Century Montreal», Shared Spaces/Partage de l'espace, 6 (Montréal, Université McGill, Département de géographie, 1986), 25 p.; S. Hertzog et R. D. Lewis, «A City of Tenants: Home Ownership and Social Class in Montreal, 1847-1881", The Canadian Geographer/ Le géographe canadien, 30,4 (1986): 316-323; G. Lauzon, Habitat ouvrier et révolution industrielle: le cas du village St-Augustin (Montréal, RCHTQ, coll. «Études et documents», no 2, 1989), $209 \mathrm{p}$.

L. Dechêne, Habitants et marchands de Montréal au XVIIe siècle (Paris et Montréal, Plon, 1974), 377-378, 488; M. Trudel, Montréal. La formation d' une société, 1642-1663 (Montréal, Fides, 1976), 46-55, 163-203.

${ }_{10}$ M. Lafrance et D.-T. Ruddel, «Physical Expansion and Socio-Cultural Segregation in Quebec City, 1765-1840», A. F. J. Artibise et G. A. Stelter, Shaping the Urban Landscape. Aspects of the Canadian City-Building Process (Ottawa, Carleton University Press, «Carleton Library Series», no 125, 1982), 149; Y. Desloges, Une ville de locataires, Québec au XVIIIe siècle (Québec, Parcs Canada, 1985), 198-199, manuscrit classé. J. Hare, M. Lafrance et D.-T. Ruddel, Histoire de la ville de Québec, 1608-1871 (Montréal, Boréal/Musée canadien des Civilisations, 1987), 39-57, 63. 
tent de dépasser l'approche traditionnelle ${ }^{11}$. L'analyse systématique du tissu urbain a permis notamment de préciser la spécialisation socioprofessionnelle par rues, quartiers et faubourgs, mais certains auteurs n'y discernent pas «un pattern très clair» et croient que «dans l'ensemble le cloisonnement n'est pas très marqué» ${ }^{12}$. L'exploitation du terrain à l'aide du bail d'héritage ou de la rente constituée a aussi fait l'objet d'une recherche $^{13}$. La question de la location immobilière à court terme a également attiré l'attention d'un chercheur, mais les résultats de ses travaux n'ont pas été publiés ${ }^{14}$.

Du côté de l'historiographie américaine des villes pré-industrielles, la stratification sociale apparaît comme l'un des thèmes majeurs. Elle est à l'origine de débats importants sur la périodisation qui remontent à la thèse égalitariste de Tocqueville et que les historiens canadiens ont intérêt à connaître. Ils tournent autour de deux questions essentielles. À quel moment l'inégalité de la richesse apparaît-elle dans les villes? À quel moment cette inégalité s'inscrit-elle dans le paysage urbain? Tel est le noeud du problème qui oppose certains historiens de la ville pré-industrielle aux historiens de la ville industrielle. Les premiers soutiennent qu'au cours du XVIIIe siècle les villes passent de l'égalitarisme et de l' «intégration socio-économique» (marchands, artisans et journaliers - riches et pauvres - qui habitent et travaillent côte à côte) à la stratification sociale et à la différenciation spatiale ${ }^{15}$. Pour

11 J. M. LeMoine, Picturesque Quebec: a Sequel to Quebec Past and Present (Montréal, Dawson Brothers, 1882), 535 p.; A. Jobin, La petite histoire de Québec (Québec, Institut St-Jean Bosco, 1948), 366 p.; G. A. Reid, The Development and Importance of the Town of Quebec 1608 1760 , thèse de doctorat, Université McGill, 1950, $358 \mathrm{p}$.

12 L. Dechêne, "Quelques aspects de la ville de Québec au XVIIIe siècle d'après les dénombrements paroissiaux», Cahiers de géographie du Québec, 28,75 (décembre 1984): 498-500.

13 L. Dechêne, "La rente du faubourg Saint-Roch à Québec, 1750-1850", RHAF, 34,4 (mars 1981): 569-596.

14 Voir Yvon Desloges, op. cit. Cette étude, traite de l'évolution du marché du logement locatif de 1690 à 1759 à l'aide, entre autres sources, d'un peu plus de 1300 baux passés devant notaire et scrute notamment la structure professionnelle des propriétaires et des locataires, les relations entre les parties, les clauses juridiques des baux, les types de logis rencontrés, la géographie de la location et l'évolution des loyers; en cela, elle s'apparente à notre mémoire de maîtrise. Desloges y effectue d'épineux calculs à partir du nombre des ménages propriétaires pour estimer la proportion des locataires: «selon les aveux et dénombrements rédigés entre 1733 et 1744, il y aurait 422 immeubles... Ces immeubles se partagent entre 328 propriétaires distincts, incluant une quarantaine de successions. En rapprochant ce dernier nombre de celui des chefs de ménage recensés par le curé Jacrau (soit 1058 ), 31\% des chefs de ménage seraient propriétaires à Québec vers $1744 \ldots$ En reportant au prorata les propriétaires des immeubles manquants, il y aurait environ 139 propriétaires de plus, ce qui donnerait une proportion maximale de $44 \%$ de propriétaires... Québec compterait donc vers 1740 au minimum $31 \%$ de propriétaires et au maximum 44\%.» (p. 185).

15 C. Bridenbaugh, Cities in the Wilderness. The First Century of Urban Life in America 1625-1742 (New York, A. A. Knopf, 1960 [1938]), 478; Cities in Revolt: Urban Life in America, 1743-1776 (New York, Oxford University Press, 1971 [A. Knopf, 1955]); J. A. Henretta, «Economic Development and Social Structure in Colonial Boston», William and Mary Quarterly, 22 (1965): 75-92; J. T. Main, The Social Structure of Revolutionary America (Princeton, Princeton University Press, 1965), 270, 286; «Trends in Wealth Concentration Before 1860», Journal of Economic History, 31 (1971): 445-447; A. Kulikoff, «The Progress of Inequality in Revolutionary Boston», William and Mary Quarterly, 28 (1971): 375-412; K. A. Lockridge, "Social Change 
les seconds, ce passage ne s'accomplit pas avant le tournant du XIXe siècle et résulte de la transformation de l'économie à la suite de l'industrialisation ${ }^{16}$. Toutefois, ni l'une ni l'autre de ces approches n'est entièrement satisfaisante.

En effet, certains auteurs insistent sur une inégalité faible mais significative caractérisant toute la période pré-révolutionnaire ${ }^{17}$. Tandis que d'autres montrent que dès la fin du XVIIe siècle la stratification sociale est déjà bien visible dans les villes de Boston, New York et Philadelphie qui comptent alors au plus quelque 6000 habitants $^{18}$. Ces derniers historiens observent une hausse phénoménale de la proportion de locataires au cours du XVIIIe siècle, passant de 30 à $80 \%$, ce qui augmente la valeur foncière dans le centre des villes et contribue à repousser les pauvres en périphérie ${ }^{19}$. Par ailleurs, des études portant sur la répartition cartographiée des taxes et des rentes foncières et immobilières et sur la spécialisation socio-professionnelle par rues et quartiers indiquent des patterns résidentiels clairement différenciés dès le XVIIIe siècle ${ }^{20}$.

Ces recherches récentes corroborent certains travaux théoriques qui soutiennent que la formation des villes américaines est marquée par une différenciation de l'espace très précoce. Dans les années 1950, Gideon Sjoberg a présenté un modèle concentrique de la structure urbaine, avec un centre riche, une zone intermédiaire à revenus moyens

and the Meaning of the American Revolution», Journal of Social History, 6 (été 1973): 403-439; G. B. Nash, «Urban Wealth and Poverty in Pre-Revolutionary America», Journal of Interdisciplinary History, 6 (1976): 545-584; «Poverty and Poor Relief in Pre-Revolutionary Philadelphia», William and Mary Quarterly, 33 (1976): 3-30; «The Social Evolution of Preindustrial American Cities, 1700-1820: Reflections and New Directions», Journal of Urban History, 13,2 (février 1987): 124.

16 S. B. Warner, Streetcar Suburbs: the Process of Growth in Boston (1870-1900) (Cambridge, Harvard University Press, 1978 [1962]); The Private City: Philadelphia in Three Periods of Its Growth (Philadelphia, 1968), 10-11; T. Hershberg, ed., Philadelphia: Work, Space, Family, and Group Experience in the Nineteenth Century (New York etOxford, Oxford University Press, 1981), 121-123, 164, 472-473.

Voir G. B. Warden, «Inequality and Instability in Eighteenth-Century Boston: a Reappraisal», Journal of Interdisciplinary History, 6 (printemps 1976): 585-620; J. G. Williamson et P. H. Lindert, American Inequality: a Macroeconomic History (New York, Academic Press, 1980), 10-11.

18 G. B. Nash, The Urban Crucible. The Northern Seaports and the Origins of the American Revolution (Cambridge, Harvard University Press, 1986 [1979]), 6-12.

${ }_{19}$ G. B. Nash, «The Social Evolution of Preindustrial American Cities, 1700-1820», R. A. Mohl, ed., The Making of Urban America (Wilmington, Scholarly Resources Books, 1988), 34-35.

20 S. Blumin, «Mobility and Change in Ante-Bellum Philadelphia», S. Thernstrom et R. Sennett, Nineteenth-Century Cities. Essay in New Urban History (New Haven, Yale University Press, 1976 [1969]), 187-188; A. Kulikoff, op. cit., 396-398; S. Blumin, The Urban Threshold: Growth and Change in a Nineteenth-Century American Community (Chicago et Londres, The University of Chicago Press, 1976), 110-111; B. Blackmar, «Re-Walking the «Walking City»: Housing and Property Relations in New York City, 1780-1840», Radical History Review, 21 (automne 1979): 131-148; S. V. Salinger et C. Wetherell, «Wealth and Renting in Prerevolutionary Philadelphia», Journal of American History, 71,4 (mars 1985): 826-840. 
et une périphérie pauvre ${ }^{21}$. James Vance a offert un modèle d'évolution des centre-villes en sept phases comprenant une phase d'exclusion (qui suit de peu la fondation et précède la phase de ségrégation) où un mécanisme de gradation des rentes immobilières urbaines (incluant les loyers) s'installe rapidement et exclut du noyau urbain les économiquement faibles $^{22}$. Ces deux modèles et les travaux empiriques qui les appuient fournissent un cadre de référence intéressant pour l'analyse et la compréhension des sociétés urbaines pré-industrielles en général et de la société montréalaise en particulier. Nous y reviendrons.

\section{2 - LES SOURCES}

Les archives disponibles pour l'étude de Montréal, contrairement à celles de Québec, n'offrent aucun recensement permettant d'embrasser directement la structure socio-professionnelle et sa localisation dans l'espace. On peut toutefois contourner partiellement ce problème en recourant à d'autres sources et en tirant parti de leur complémentarité.

Le terrier de Montréal tenu par les Sulpiciens, seigneurs de l'île, enregistre les mutations des emplacements urbains intra-muros de 1666 à 1795 avec le nom des propriétaires fonciers. Il comprend un plan découpé en dix planches qui rend possible la localisation précise des emplacements dans l'espace urbain ${ }^{23}$. L'aveu et dénombrement de la seigneurie dressé par les Sulpiciens en 1731 s'est aussi avéré utile. Il fournit les noms et prénoms des propriétaires ainsi que les matériaux des immeubles. En découpant le document selon les secteurs de la ville, nous avons pu localiser plusieurs immeubles locatifs et, partant, les ménages locataires qui les occupent ${ }^{24}$.

Le «recensement» de 1741 effectué par la Compagnie des Indes nous fut, quant à lui, précieux ${ }^{25}$. Il renseigne d'abord sur les noms,

21 G. Sjoberg, «The Preindustrial City», American Journal of Sociology, 60 (1955): 438445; The Preindustrial City: Past and Present (New York, Free Press of Glencoe, 1960).

22 Voir J. E. Vance, «Focus on Downtown», L. S. Bourne, ed., The Internal Structure of the City. Readings on Space and Environment (Toronto, Oxford University Press, 1971), 114115 .

23 Société historique de Montréal, «Livre Terrier de la seigneurie de Montréal mentionnant les concessions et mutations de terrains compris dans les limites des anciennes fortifications», Les origines de Montréal (Mémoires de la Société historique de Montréal, 11e livraison, Montréal, Adj. Ménard, imprimeur et éditeur, 1917), 40ss.

${ }_{24}$ «Aveu et Dénombrement de messire Louis Normand, prêtre du Séminaire de SaintSulpice à Montréal, au nom et comme fondé de procuration de messire Charles-Maurice Le Pelletier, supérieur du Séminaire de Saint-Sulpice de Paris, pour la seigneurie de l'île de Montréal» (septembre 1731), Rapport des Archives nationales du Québec (RAPQ) (1941-42): 1-76.

25 En réalité, il s'agit de procès-verbaux de perquisitions que nous utilisons en guise de recensement. En effet, en 1741 , la Compagnie des Indes procède à des perquisitions afin de préserver son monopole sur l'exportation de castor du Canada et l'importation de marchandises fabriquées hors de la Nouvelle-France. Menacée par le trafic clandestin avec les colonies anglaises, la Compagnie des Indes obtient la permission de visiter les magasins et les résidences privées «afin de confisquer les dites marchandises et de les brûler publiquement». D'ailleurs, le manuscrit porte le titre «Recensement fait en cette ville par la Compagnie des Indes pour les indiennes et 
prénoms et patronymes des locataires et pensionnaires. Dans la version publiée, les annotations de E.-Z. Massicotte enrichissent le document en précisant les données nominatives, en fournissant occasionnellement la profession des individus, et, surtout, en indiquant le renvoi avec référence exacte au terrier de Montréal et à son plan. Le recensement de 1741 permet donc de calculer la proportion des locataires et, grâce aux notes ajoutées, de replacer aisément ceux-ci dans l'espace urbain. Aussi l'avons-nous utilisé comme point de départ de notre enquête, pour cartographier la répartition des locataires montréalais.

Enfin, le bail à loyer et prix d'argent constitue un élément très important de notre corpus. Il fournit pour chaque cas de location le loyer annuel, les renseignements d'ordre foncier et immobilier comme la rue, le quartier ou le faubourg, ainsi que l'identité des bailleurs et des locataires, c'est-à-dire leurs noms, prénoms, patronymes et généralement leurs métiers quoique nous ayons parfois dû recourir aux Inventaires des greffes des notaires pour compléter ces renseignements. Par recoupement des données nominatives avec l'une ou l'autre des sources déjà mentionnées, cette source permet de connaître la composition socio-professionnelle des locataires montréalais et leur distribution sur le quadrillage urbain ${ }^{26}$.

Notre étude repose donc sur un cumul de sources négligées par la recherche actuelle, en histoire urbaine, qui apportent un éclairage neuf sur la société montréalaise du XVIIIe siècle. Cette analyse des locataires, qui se présente comme une étude de cas d'une ville pré-industrielle, fait appel à une problématique en deux volets: premièrement, cerner la relation entre la structure sociale et l'espace physique urbain et, deuxièmement, établir une comparaison entre villes pré-industrielles et industrielles quant à la différenciation de l'espace. À la suite des travaux canadiens et américains mentionnés ci-haut, cet article participe à la réévaluation des thèses «égalitaristes» et «intégrationnistes» encore en cours et cherche à offrir aux historiens de la ville industrielle quelques points d'ancrage dans le passé.

\section{3 - MONTRÉAL EN 1731-1741: TABLEAU D'ENSEMBLE}

On sait déjà qu'en 1731 Montréal est une petite ville de frontière dont l'économie repose en grande partie sur le commerce des fourrures. Elle compte alors près de 3000 habitants, faubourgs compris ${ }^{27}$. En

autres effets prohibés pour être marqués en vertu de l'ordonnance de messieurs le Gouverneur et Intendant». E.-Z. Massicotte, «Un recensement inédit de Montréal en 1741», Mémoires de la Société royale du Canada, série III, 15 (mai 1921): 1-61.

26 Ont été dépouillés, pour la période 1731-1741, les greffes des notaires suivants: J.-B. Adhémar, L.-C. Danré de Blanzy, F. Lepailleur, C.-J. Porlier, J.-C. Raimbault, F. Simonnet, C. $-\mathrm{R}$. Gaudron de Chèvremont. Un total de 153 baux ont été recueillis.

27 Estimation de L. Dechêne, «La croissance de Montréal au XVIIIe siècle», RHAF, 27,2 (septembre 1973): 164. 
1741 elle passe à environ 3575 âmes réparties en 511 ménages (voir tableau 1), soit presque sept personnes par ménage. À cette date, les faubourgs demeurent peu développés et la ville intra-muros accapare encore $86 \%$ des ménages. La croissance de 1731 à 1741 , lente mais continue, masque la mobilité d'une population où les arrivées et les départs semblent fréquents, du moins chez les locataires.

D'après le recensement de 1741 , les locataires montréalais représenteraient $35 \%$ des ménages qui habitent la ville intra-muros (tableau 1). Mais, deux indices laissent croire à un sous-dénombrement des locataires. D'abord, onze locataires dont les baux étaient en vigueur au cours de l'année 1741 ne sont pas mentionnés dans le recensement. Ensuite, dans la version du recensement qu'il a publiée, E.-Z. Massicotte commente le cas du ménage Charlu-Boulard, des locataires, que les recenseurs avaient omis d'inscrire ${ }^{28}$. Ainsi, nous pouvons raisonnablement supposer que le pourcentage de locataires en 1741 était supérieur à $35 \%$.

\section{TABLEAU 1}

Distribution de l'ensemble des ménages et des ménages locataires sur le plan urbain montréalais

1741

Ensemble des ménages

Ville intra-muros

439

Ménages locataires Nombre

153

$\%$

Faubourgs

Saint-Joseph

Saint-Louis

Sainte-Marie

Total

$\begin{array}{rrr}49 & 12 & 24,5 \\ 15 & 2 & 13,3 \\ 8 & 1 & 12,5 \\ 511 & 168 & 32,9\end{array}$

Sources: E.-Z. Massicotte, «Un recensement inédit de Montréal en 1741», Mémoires de la Société royale du Canada, 15 (mai 1921): 1-61.

\section{4 - LA CARTOGRAPHIE DES LOCATAIRES}

La carte 1 montre la répartition des locataires dans la ville intramuros d'après le recensement de 1741. Cette carte et celles qui vont suivre présentent un quadrillage qui découpe les artères principales, c'est-à-dire les rues Saint-Paul, Notre-Dame et Saint-Jacques, identifiées respectivement par les lettres $\mathrm{X}, \mathrm{Y}$ et $\mathrm{Z}$, en tronçons de longueurs équivalentes identifiés par des chiffres allant de 1 à 7 ; les rues perpendiculaires n'ont pas subi de découpage et sont simplement identifiées

28 E. -Z. Massicotte, «Un recensement...», no 119, 22-23. 


\section{CARTE 1}

Localisation des ménages locataires d'après le recensement de 1741

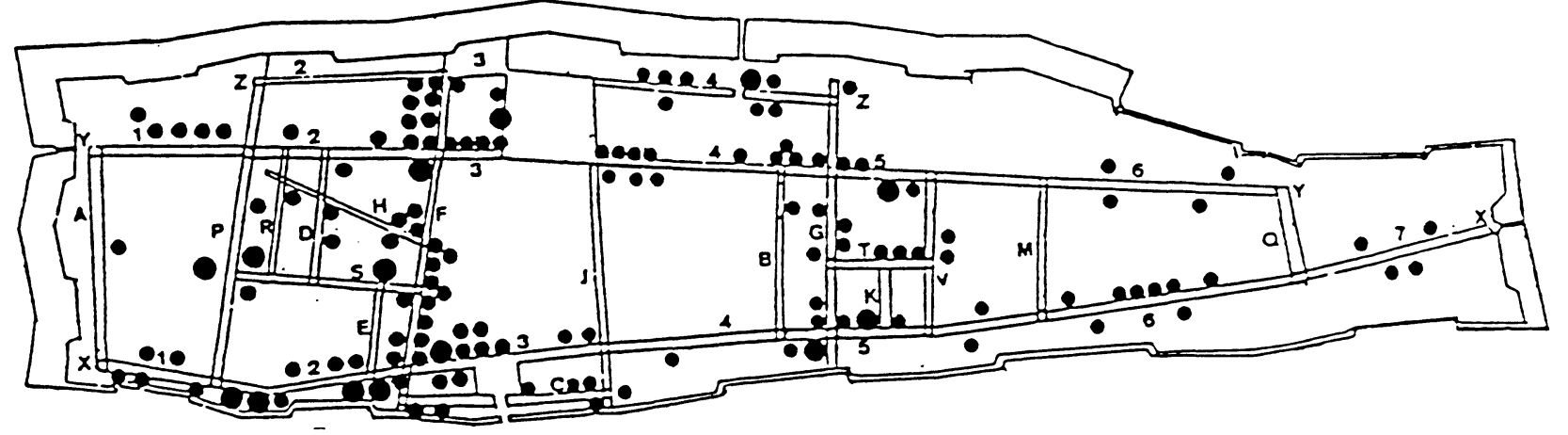

\section{LÉGENDE}

A- Saint-Augustin

B- Saint-Jean-Baptiste

C- Capitale

D- Saint-Jean

E- Saint-Éloy

F- Saint-François

G- Saint-Gabriel

H- de L'Hôpital

J- Saint-Joseph

K- Saint-Denis

M- Saint-Charles

P- Saint-Pierre

Q- Notre-Dame de Bonsecours

- immeubles à un seul logis locatif

immeubles à plusieurs logis locatifs.
R- Saint-Alexis

S- Saint-Sacrement

T- Sainte-Thérèse

V- Saint-Vincent

X- Saint-Paul

1- de Saint-Augustin à Saint-Pierre

2- de Saint-Pierre à Saint-François

3- de Saint-François à Saint-Joseph

4- de Saint-Joseph à Saint-Jean-Baptiste

5- de Saint-Jean-Baptiste à Saint-Vincent

6- de Saint-Vincent à Bonsecours

7- de Bonsecours à la porte Sainte-Marie
Y- Notre-Dame

1- de Saint-Augustin à Saint-Pierre

2- de Saint-Pierre à Saint-François

3- de Saint-François à Saint-Joseph

4- de Saint-Joseph à Saint-Jean-Baptiste

5- de Saint-Jean-Baptiste à Saint-Vincent

6- de Saint-Vincent à Bonsecours

Z- Saint-Jacques

2- de Saint-Pierre à Saint-François

3- de Saint-François à Saint-Joseph

4- de Saint-Joseph à Saint-Gabriel 
par une lettre empruntée si possible au nom de la rue (ex.: A = SaintAugustin, $\mathrm{C}=$ Capitale, $\mathrm{H}=$ de l'Hôpital, $\mathrm{V}=$ Saint-Vincent, etc.)

Si la carte 1 montre un certain éparpillement des locataires dans l'espace urbain, plusieurs rues se démarquent néanmoins avec une concentration des ménages locataires, perceptible dans le rapport entre la proportion de logis locatifs et le nombre de maisons. Ainsi, en ne retenant que les bouts de rues comptant $40 \%$ et plus de logis locatifs sur un bâti d'au moins 11 maisons, nous rencontrons au sud-ouest, près de la porte du port, la rue Saint-Paul 2 et 3, qui représente le coeur économique de la ville. Zone mitoyenne, la rue Saint-François s'affirme également par sa concentration de locataires. À la périphérie nord-ouest, formant une troisième zone, on retrouve les rues Notre-Dame 5 et SaintJacques 4 . En moyenne, pour ces cinq bouts de rues, les ménages locataires comptent pour près de $50 \%$ de l'ensemble des ménages. Ce coup d'oeil rapide de la répartition des locataires permet de circonscrire trois zones urbaines. Retenons ce schéma simple car il accompagne tout au long notre analyse.

Pour la ville intra-muros, 153 baux de location ont été retrouvés. Le profil de la répartition des locataires à partir de ces actes (carte 2) épouse assez bien celui du recensement de 1741 (carte 1). On observe une concentration de locataires sur les rues Saint-Paul 2 et 3, et SaintFrançois pour lesquelles nous disposons d'autant de baux qu'il y a de ménages locataires au recensement de 1741. Toutefois, deux bouts de rues à forte concentration de locataires, Saint-Jacques 4 et Notre-Dame 5 , sont sous-représentés dans les baux.

\section{5 - RÉPARTITION DES GROUPES SOCIO-PROFESSIONNELS}

L'analyse des catégories socio-professionnelles chez les locataires montre trois groupes principaux différenciés par les niveaux de loyer. Les plus aisés comptent pour 37,8\% des cas de location repérés de 1731 à 1741 (voir graphique 1). Sans doute s'agit-il pour la plupart de jeunes marchands au début de leur carrière pour lesquels l'achat d'une propriété n'est pas encore accessible ou d'officiers militaires poursuivant une carrière où les changements d'affectation sont nombreux. Les gens de métier représentent près de la moitié des effectifs, mais leur proportion réelle sur le marché est sans doute plus élevée en raison des ententes orales prises de gré à gré dont le nombre demeure indéterminé ${ }^{29}$.

La répartition selon les tranches de loyer permet d'observer une différence appréciable quant aux sommes versées par chacun de ces deux groupes. Retenons pour l'instant que si $86,5 \%$ des ménages des

29 Voir J.-F. Leclerc, «Aspects des relations sociales en Nouvelle-France: les voies de fait dans la juridiction de Montréal au XVIIIe siècle», mémoire de maîtrise (histoire), Université de Montréal, 1985. L'auteur fait état de mésententes survenues entre propriétaires et locataires en l'absence de bail écrit. 


\section{CARTE 2}

Localisation des ménages locataires

d'après les baux notariés, 1731-1741

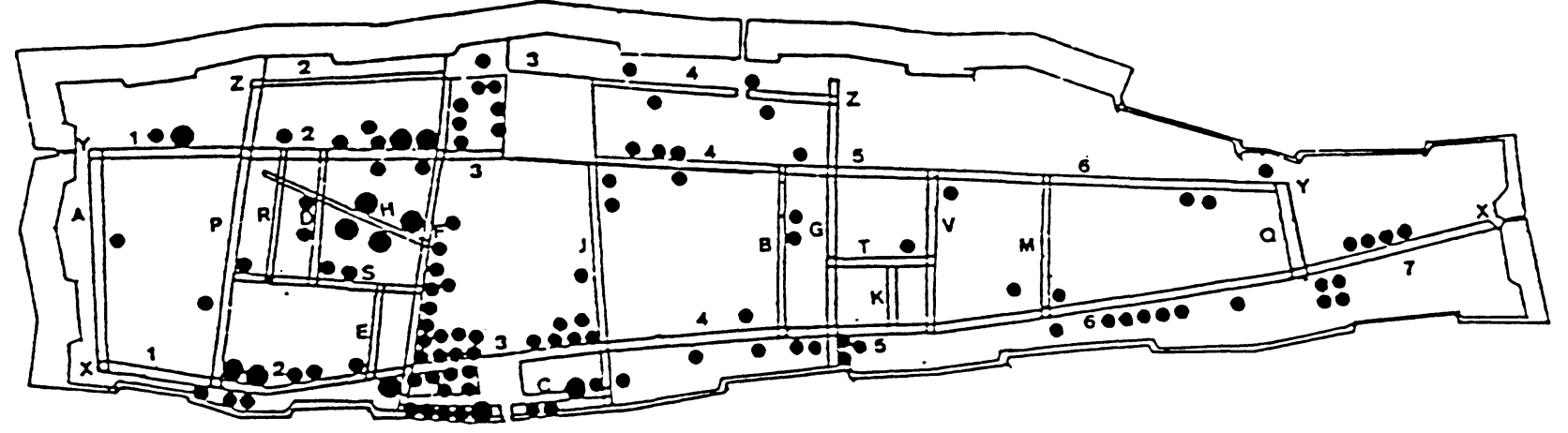

Voir la légende de la carte 1.

- Logis locatifs dont un seul bail fut retrouvé.

Logis locatifs dont plusieurs baux furent retrouvés. 


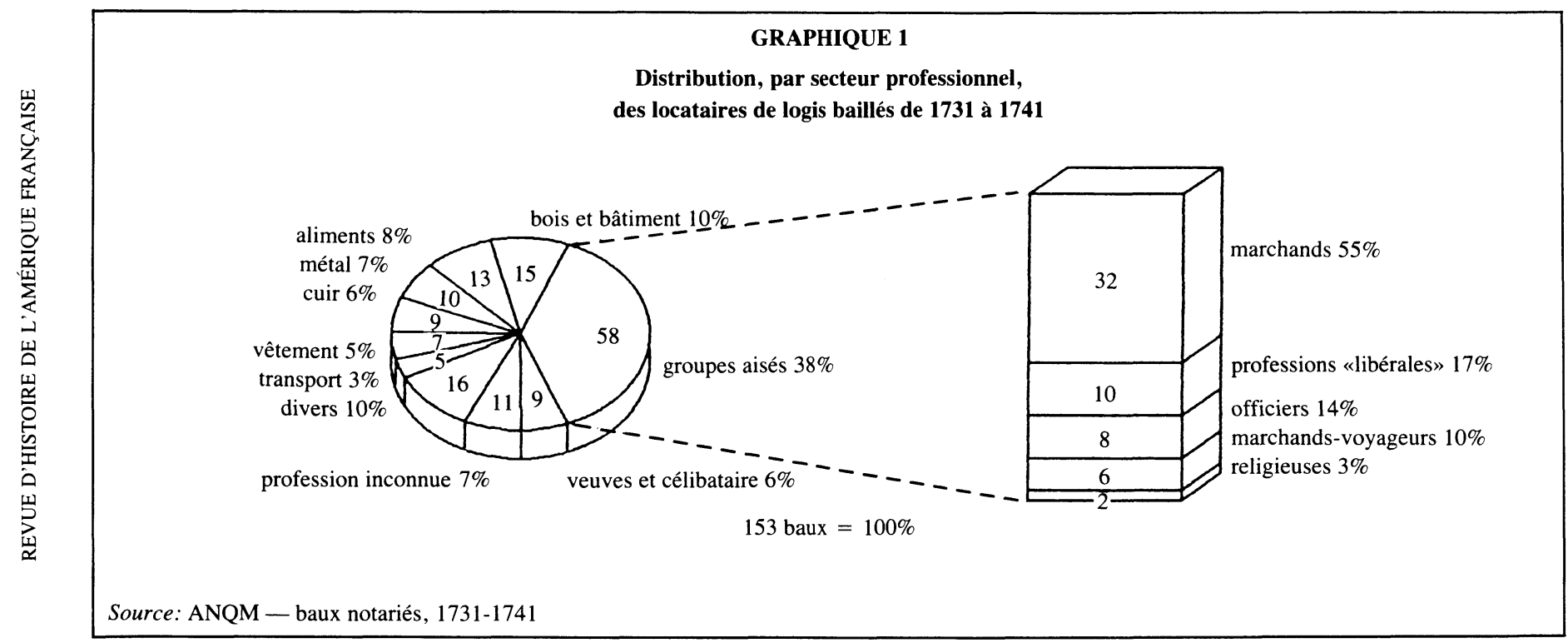

$N$ 
gens de métier paient 150 livres et moins annuellement, 84,7\% des ménages aisés paient entre 100 et 600 livres. L'analyse qui suit utilise ces écarts dans la valeur des loyers comme un indice de la distribution de la richesse et du bien-être des groupes composant cette société urbaine

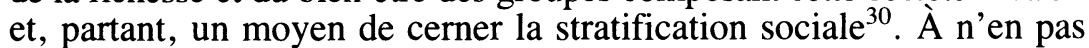
douter, les revenus disponibles influencent les choix résidentiels car ils fixent bien souvent le niveau du loyer, la superficie du logement et les qualités physiques des lieux, soit trois variables qui vont déterminer la localisation dans l'espace urbain.

Notre analyse va permettre non seulement de mieux circonscrire le clivage entre groupes aisés et groupes populaires, mais aussi d'apprécier la disparité endogène de chacun. Elle va également identifier un sous-groupe, plus défavorisé au plan des conditions de logement: les gens de profession inconnue mais aussi et surtout les femmes, les veuves en particulier, pour lesquelles peu de baux ont été retrouvés.

\section{A - Les locataires aisés}

Les baux de location montrent que ceux-ci paient des loyers qui représentent 1,7 fois la valeur locative défrayée par les groupes populaires car leur loyer médian atteint les 180 livres. Le clivage est peutêtre faible, mais il est significatif. Toutefois il y a des différences importantes au sein même de ce groupe entre les marchands et les officiers militaires d'une part et les autres professions d'autre part. Alors que les négociants ${ }^{31}$ paient un loyer médian de 220 livres, les officiers des troupes de la Marine, de 180 livres et les marchands-voyageurs, de 150 livres, les chirurgiens, huissiers et notaires, quant à eux, défraient un loyer médian de 105 livres $^{32}$. Pour ce qui a trait au type de logis, $82 \%$ des ménages aisés choisissent une maison unifamiliale et neuf fois sur dix, elle est en pierre.

La répartition des lieux de résidence de l'élite montréalaise sur le territoire n'est pas aléatoire comme le montre la carte 3 . Elle se concentre spécifiquement dans les rues Saint-Paul 3 et Notre-Dame 2 où elle occupe respectivement 71 et $92 \%$ des logis locatifs baillés au cours de la période. Les marchands forment la majorité du groupe. On retrouve aussi ces derniers en moindre proportion sur la rue Capitale où ils voisinent avec les auberges et les cabarets. On remarque également une

30 Plusieurs auteurs sont d'avis que la valeur des rentes immobilières est un meilleur indice de la richesse des ménages que les salaires. Voir S. V. Salinger et C. Wetherell, op. cit., 827828 .

31 Parmi les marchands, nous avons une femme qui se déclare marchande, M. Fayette de La Fayette, qui loue une maison de pierre à 2 étages sur la rue Saint-Paul 3 pour 150 livres. Archives nationales du Québec à Montréal (ANQM), F. Lepailleur, 14 août 1737.

32 Nous incluons dans cette catégorie supérieure les religieuses Hospitalières de SaintJoseph qui louent deux logis: un de 100 livres et l'autre de 170 livres. ANQM, J.-Bte Adhémar, 11 décembre 1731; J.-C. Raimbault, 5 octobre 1733. 


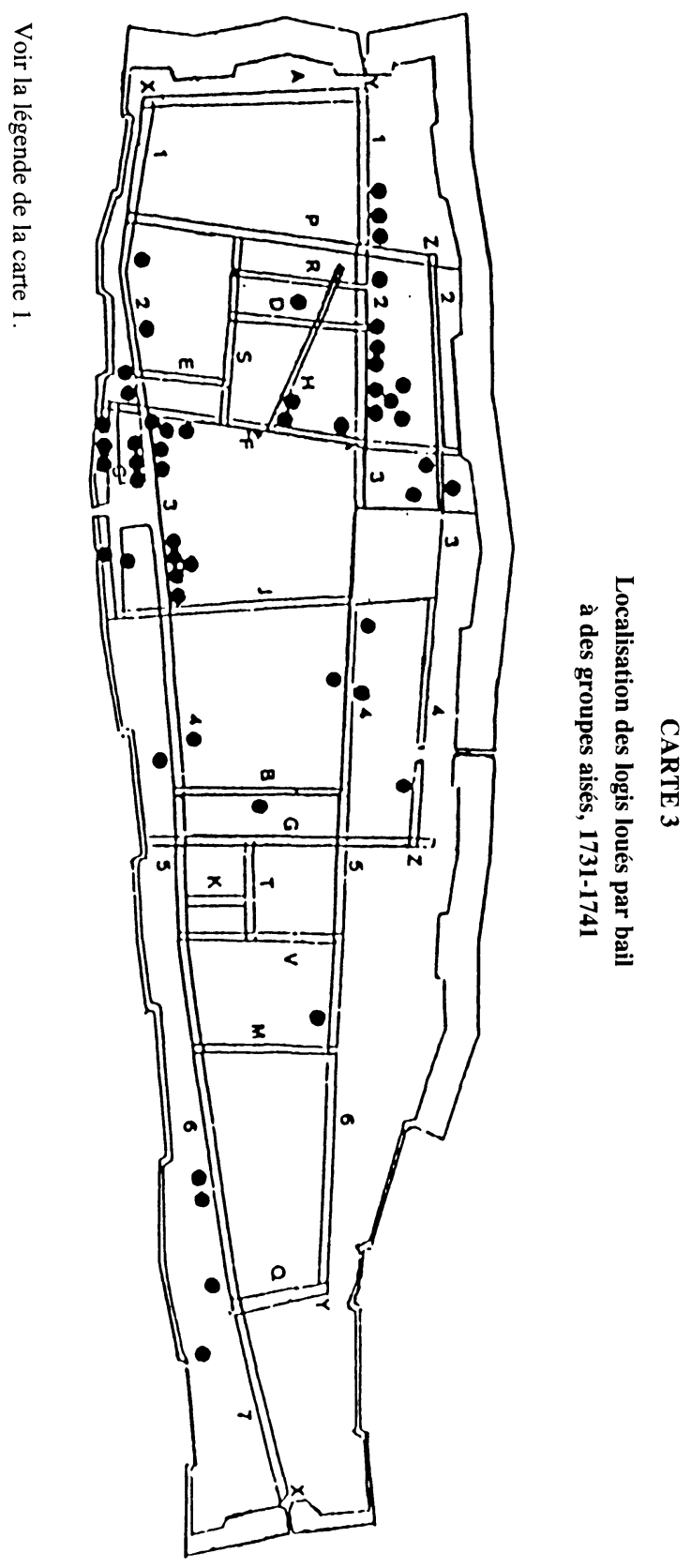


concentration particulière sur la rue St-Paul 6 des officiers de plume et d'épée et autres ménages appartenant à la noblesse et cela, tant chez les locataires que chez les bailleurs ${ }^{33}$. La répartition des locataires aisés met donc en relief la concentration des activités commerciales dans l'espace urbain.

\section{B - Les locataires issus des milieux populaires}

\section{Les gens de métier}

D'après les baux, les gens de métier paient un loyer médian annuel de 105 livres et, bien qu'ils préfèrent louer des maisons unifamiliales - en bois pour les deux tiers du groupe - beaucoup ne peuvent louer qu'une partie de maison, soit $38 \%$. Ce portrait doit cependant être nuancé car le groupe n'est pas homogène. Si les gens du transport (charretier, voiturier, navigateur) paient 90 livres de loyer annuel moyen, il en coûte 129 livres aux artisans du cuir (essentiellement des cordonniers). Les loyers des autres gens de métier se situent entre ces deux bornes ${ }^{34}$. Un écart prononcé peut exister au sein d'une même catégorie professionnelle. Les tonneliers paient un loyer moyen très bas de 71 livres, alors que les menuisiers défraient plutôt des loyers exceptionnellement élevés pour des gens de métier, soit 177 livres en moyenne.

Pour apprécier le poids de ces loyers il faudrait connaître le revenu de ces artisans et petits commerçants, ce qui est évidemment impossible. On peut toutefois, à défaut de revenu, utiliser les données sur les salaires en supposant que les gages versés à certains hommes de métier correspondent à peu près à ce qu'ils gagneraient en travaillant à leur compte. L'échelle des salaires des hommes de métier proposée en 1739 par l'intendant Gilles Hocquart se situe entre 300 et 400 livres par année ${ }^{35}$. Les loyers versés apparaissent fort onéreux en regard de ces chiffres. Avec un loyer médian de 120 livres pour l'ensemble de l'espace urbain $^{36}$, c'est donc dire que le logement paraît absorber de 30 à

33 En effet, sur la rue Saint-Paul 6, nous retrouvons comme propriétaires: Noyan, Pecaudy de Contrecoeur, Rocbert de La Morandière, la veuve Testard de Montigny, Vaudreuil; comme locataires: Ailleboust de Manthet des Muceaux, la veuve Chapte de la Corne, Daneau De Muy, la veuve Le Ber de Senneville, Le Moyne de Longueuil, la veuve Ramezay, Saint-Ours, la veuve Testard De Montigny, le gouverneur de la ville Dubois Berthelot de Beaucourt, Chaussegros De Léry et Madame Bégon. Le Roi loue le château de Vaudreuil pour servir de résidence temporaire au gouverneur de la colonie, le marquis de Beauharnois et à l'intendant durant leurs séjours à Montréal. Voir Pehr Kalm, op. cit., 201, 521; E.-Z. Massicotte, op. cit., 32-36; ANQM, F. Lepailleur, 3 août 1733, 2 septembre 1735; J.-C. Raimbault, ler août 1737; F. Simonnet, 14 juin 1740 .

34 Bâtiment et bois (briquier, chaisier, charpentier, maçon, menuisier, tonnelier), 100 livres; vêtements (chapelier, tailleur d'habits), 108 livres; métaux (armurier, forgeron, taillandier), 115 livres; alimentation (aubergiste, boucher, cabaretier), 127 livres; la catégorie divers regroupe les agriculteurs, arpenteur, charron, orfèvres et perruquiers.

35 Hocquart au Ministre, Québec, 22 septembre 1739, AC, C11A, vol. 71, 123. Cité par C. Nish, Les bourgeois-gentilshommes de la Nouvelle-France, 1729-1748 (Ottawa, Fides, 1968), 40.

36 Il s'agit bien du loyer médian calculé à l'aide des baux de location notariés (loyer moyen: 150 livres). Nous nous gardons de proposer ces mesures de tendances centrales comme équivalant aux loyers moyen et médian réels puisqu'ils ne tiennent pas compte des ententes verbales. 
$40 \%$ du salaire annuel du chef de famille appartenant aux groupes populaires. Prenons à titre d'exemple, le cas de Théophile Barthe dit Bardet, armurier du roi, qui loue pour trois ans une maison unifamiliale, rue Saint-François, à raison de 170 livres par année alors que son salaire est de 480 livres $^{37}$. Si ce salaire est le seul revenu du ménage, ce qui est vraisemblable, le logement représenterait environ $35 \%$ d'un budget que Barthe doit aussi employer pour nourrir et vêtir sa femme et ses sept enfants ${ }^{38}$.

Si un artisan utilise une telle portion de son salaire pour se loger, on peut aisément supposer que le pourcentage est plus élevé encore pour les journaliers. Bref, la ponction locative sur le budget des groupes populaires urbains à l'époque de la Nouvelle-France semble particulièrement importante ${ }^{39}$.

Dans l'espace urbain intra-muros, les gens de métier préfèrent les rues Saint-François, Saint-Paul 2 et de l'Hôpital (voir carte 4) où les artisans et les petits commerçants occupent respectivement 71,67 et $78 \%$ des logis locatifs baillés. Il y a même une spécialisation professionnelle dans l'une de ces rues, soit la rue Saint-François où nous retrouvons un nombre significatif de baux passés par des artisans du fer (armurier, forgeron, serrurier, taillandier) ${ }^{40}$.

La spécialisation caractérise également la rue Capitale puisque, au cours de la période, près de $40 \%$ des locataires sont des aubergistes et des cabaretiers ${ }^{41}$, prêts à accueillir les arrivants fraîchement débarqués qui franchissent la porte du port et se dirigent vers la place du marché.

Par ailleurs, on retrouve une partie des gens de métier sur la rue Saint-Paul 7, dans le «quartier Bonsecours ${ }^{42}$. Cette concentration est également significative puisque ceux-ci occupent $88 \%$ des logis loués par contrat notarié.

37 Voir C. Nish, op. cit., 40; ANQM, C.-J. Porlier, 15 novembre 1736.

38 Voir R. Bouchard, Les armuriers en Nouvelle-France (Québec, MAC, 1978), 44.

39 À titre de comparaison, rappelons une analyse de A. Sutcliffe concernant les classes ouvrières de l'Angleterre industrielle qui insiste sur la part du budget ouvrier consacré au logement et sur une augmentation de celle-ci entre 1850 et 1900-1910. Pourtant, le plus haut pourcentage rapporté dans son étude est celui de York en 1901 où les loyers atteignent une moyenne de $29 \%$ des revenus ouvriers, soit une proportion au-dessous de celle calculée ici. Voir A. Sutcliffe, «In Search of the Urban Variable. Britain in the Later Nineteenth Century», D. Fraser et A. Sutcliffe, The Pursuit of Urban History (Londres, E. Arnold, 1983), 254-255.

${ }_{40}$ ANQM, minutes des notaires J.-Bte Adhémar, 22 juillet 1741, 28 juillet 1741; F. Lepailleur, 7 juillet 1733, 20 avril 1735; C.-J. Porlier, 15 novembre 1736; F. Simonnet, 29 octobre 1739.

${ }_{41}$ ANQM, minutes des notaires L.-C. Danré de Blanzy, 11 août 1740; J.-Bte Adhémar, 13 septembre 1741 , 16 juin 1732, 9 juillet 1735; F. Lepailleur, 17 septembre 1738, 4 avril 1736 .

${ }_{42}$ Selon l'expression utilisée dans les baux pour ce secteur de la ville qui semble comprendre tout le secteur est (convention montréalaise) de la rue Saint-Jean-Baptiste. 


\section{CARTE 4}

Localisation des logis loués par bail à des gens de métier, 1731-1741

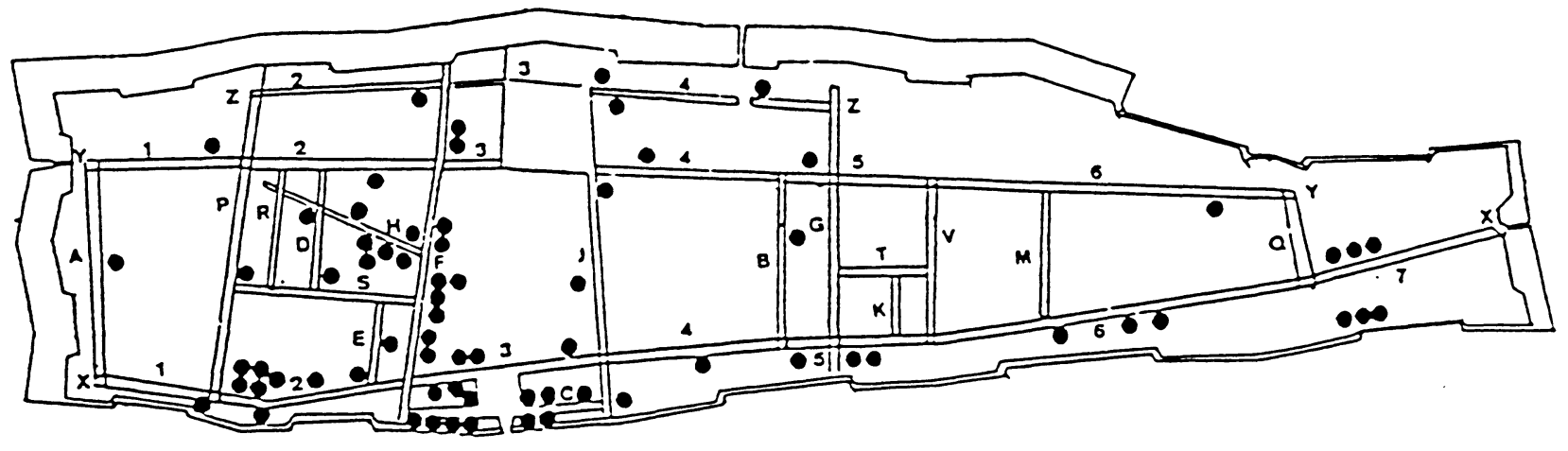

Voir la légende de la carte 1. 


\section{Les groupes défavorisés}

La pauvreté semble déjà un phénomène urbain important à cette époque si l'on en croit les différentes initiatives prises pour la contrer. Dès 1688, le Conseil souverain décide d'instaurer un Bureau des pauvres à Québec, Trois-Rivières et Montréal, destiné à soutenir les véritables démunis et à inciter les personnes aptes au travail à s'activer ${ }^{43}$. À Montréal, les Sulpiciens et l'Hôpital Général se sont également occupés de l'indigence ${ }^{44}$. Les baux fournissent quelques informations sur le phénomène.

Les gens de profession inconnue paient un loyer médian de 84 livres seulement, ce qui nous permet de croire qu'il s'agit de journaliers sans métier. Leurs gages sont certainement inférieurs à ceux que nous avons cités plus haut pour les artisans, ce qui veut dire que le loyer absorbe une proportion considérable de leurs revenus. La journée des manoeuvres vaut en général une livre dix sols dans la colonie, mais ceux-ci ne travaillent pas régulièrement. Le chômage est important en hiver. Leurs conditions de vie semblent donc particulièrement précaires et vraisemblablement ils consacrent plus de $40 \%$ de leurs revenus au logement.

Cette ponction considérable du revenu des moins nantis, comparée à la part de 30 à $40 \%$ consentie par les gens de métier, montre que du haut au bas de l'échelle sociale, le rapport entre revenu et loyer est inversement proportionnel. Alors qu'un loyer élevé représente généralement une faible proportion du revenu, un petit loyer équivaut habituellement à une fraction substantielle de ce dernier. C'est ici que la différenciation des groupes sociaux s'opère véritablement. Ce modèle mathématique apparaît comme une règle caractérisant la masse des locataires ${ }^{45}$.

Sur les 153 chefs de ménage locataires recensés en 1741, près du quart sont des femmes, soit 17 veuves et 18 femmes mariées et célibataires ${ }^{46}$. Cette forte proportion reflète-t-elle une faiblesse des moyens pécuniaires? L'étude des niveaux de loyer, à partir des baux, permet de mieux cerner cette question.

43 Voir W. H. Atherton, op. cit., 306-307; A. Lachance, «Le Bureau des pauvres de Montréal 1698-1699. Contribution à l'étude de la société montréalaise de la fin du XVIle siècle», Histoire sociale/Social History, 4 (novembre 1969): 99-110; R. Rumilly, op. cit., 234.

44 L. Roberts, op. cit., 85-86; H. Lapointe-Roy, Charité bien ordonnée. Le premier réseau de lutte contre la pauvreté à Montréal au 19e siècle (Montréal, Boréal, 1987), 30.

45 Des travaux portant tant sur les villes pré-industrielles qu'industrielles arrivent aux mêmes conclusions. Voir S. V. Salinger et C. Wetherell, op. cit., 828; A. Sutcliffe, op . cit., 254-255. Ce dernier précise qu'à York en 1901, la plus forte proportion, soit 29\%, est payée par les familles les plus pauvres, qui subsistent avec moins de 18 shillings par semaine, et la plus faible proportion, $9 \%$, par une minorité de familles prospères qui gagnent plus de $£ 3$ par semaine.

Le nom des femmes et veuves est généralement incomplet dans le recensement et si E.Z. Massicotte précise la plupart du temps le nom complet, suivi du nom de l'époux, il ne mentionne pas le métier de ce dernier, sauf exception. 
Bien que les femmes (huit veuves et une célibataire) paraissent en bonne posture financière puisqu'ensemble elles paient un loyer médian de 100 livres, il faut les départager pour mieux saisir leur condition. Ainsi, alors que les veuves rattachées au groupe aisé paient 180 livres, celles rattachées aux groupes populaires (incluant la célibataire) paient un loyer médian de 75 livres ${ }^{47}$. Le clivage social, ici, apparaitt bien nettement. De tous les groupes analysés dans notre étude, ce sont les femmes des groupes populaires qui semblent vivre les pires conditions de logement ${ }^{48}$. Les veuves, chargées de jeunes enfants, sont dans une situation très difficile, surtout si les sources de revenus se sont taries.

Par ailleurs, la distribution dans la ville des veuves et des gens de profession inconnue ou journaliers ne montre pas de concentration (carte 5). Leur éparpillement est en soi significatif. Ces groupes se logent là où ils le peuvent se contentant d'une partie de maison, souvent d'une seule pièce.

\section{6 - UNE STRUCTURE LOCATIVE À TROIS SECTEURS}

L'analyse qui précède a permis de dégager une géographie différenciée et souvent spécialisée de la location. La répartition des loyers médians (voir carte 6) et les matériaux des immeubles retrouvés sur les bouts de rues vont permettre de mieux dégager la stratification de l'espace locatif montréalais.

Commençons par la rue Capitale, où se concentrent les aubergistes, les cabaretiers et les marchands, la rue Saint-Paul 3 à laquelle nous pouvons rattacher la rue Notre-Dame 2, où les marchands forment la majorité des locataires, et la rue Saint-Paul 6 que la présence nobiliaire distingue. Ces quatre bouts de rues, dont le matériau dominant est la pierre, sont les plus coûteux de la ville avec des loyers médians respectifs de 150, 170 et 173 et 190 livres. Les locataires aisés s'installent donc principalement, sauf exception (Notre-Dame 2 et SaintPaul 6), dans le noyau portuaire. Voyons en second lieu les rues SaintFrançois, de l'Hôpital et Saint-Paul 2 qui ont une concentration d'artisans et de gens de métier avec des loyers médians de 110, 120 et 150 livres respectivement alors que leur bâti est, grosso modo, moitié de bois et moitié de pierre. Donc, les gens de métier habitent en majorité dans une frange urbaine attenant au noyau, là où les loyers sont abordables.

On retrouve enfin, en troisième lieu, la rue Notre-Dame 5 , pour laquelle un seul bail à 110 livres fut repéré, et la rue Saint-Jacques 4 où

47 C'est par la profession du mari défunt que nous classons les veuves. Nous avons placé celles dont la profession du mari reste inconnue parmi les groupes populaires.

48 Dans leur étude sur Philadelphie, Salinger et Wetherell ont aussi indiqué que les locataires les plus pauvres, c'est-à-dire ceux payant les loyers les plus bas, sont en majorité des femmes, qu'elles vivent généralement en périphérie et qu'elles sont les locataires les plus mobiles. Voir S. V. Salinger et C. Wetherell, op. cit., 835-836. 
CARTE 5

Localisation des gens de profession

inconnue et des veuves, 1731-1741

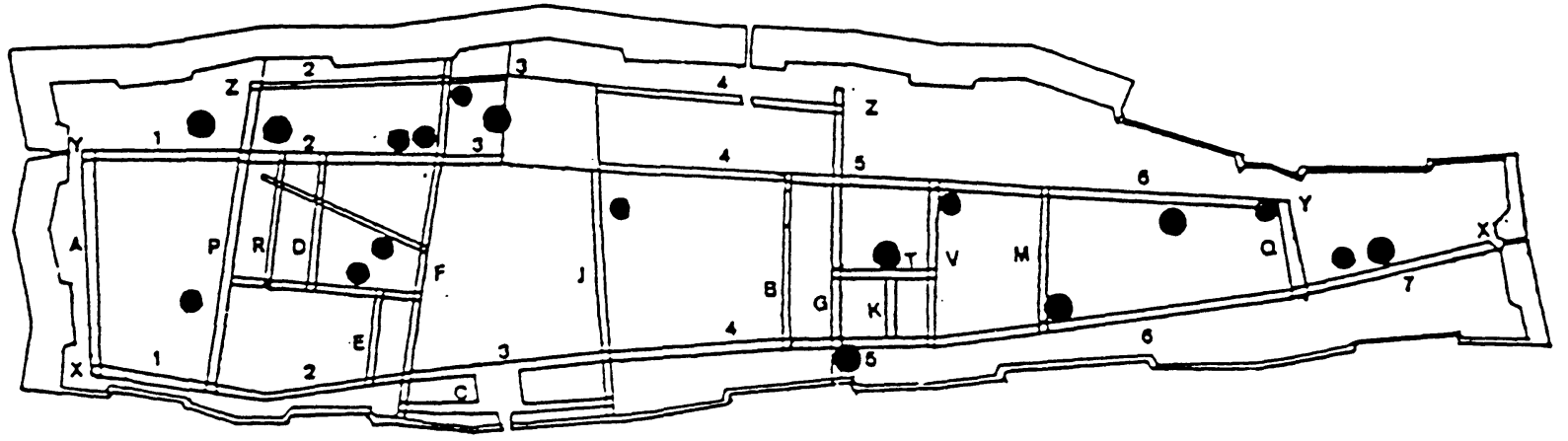

Voir la légende de la carte 1.

- profession inconnue.

- veuves. 
STRATIFICATION SOCIALE ET DIFFÉRENCIATION SPATIALE...

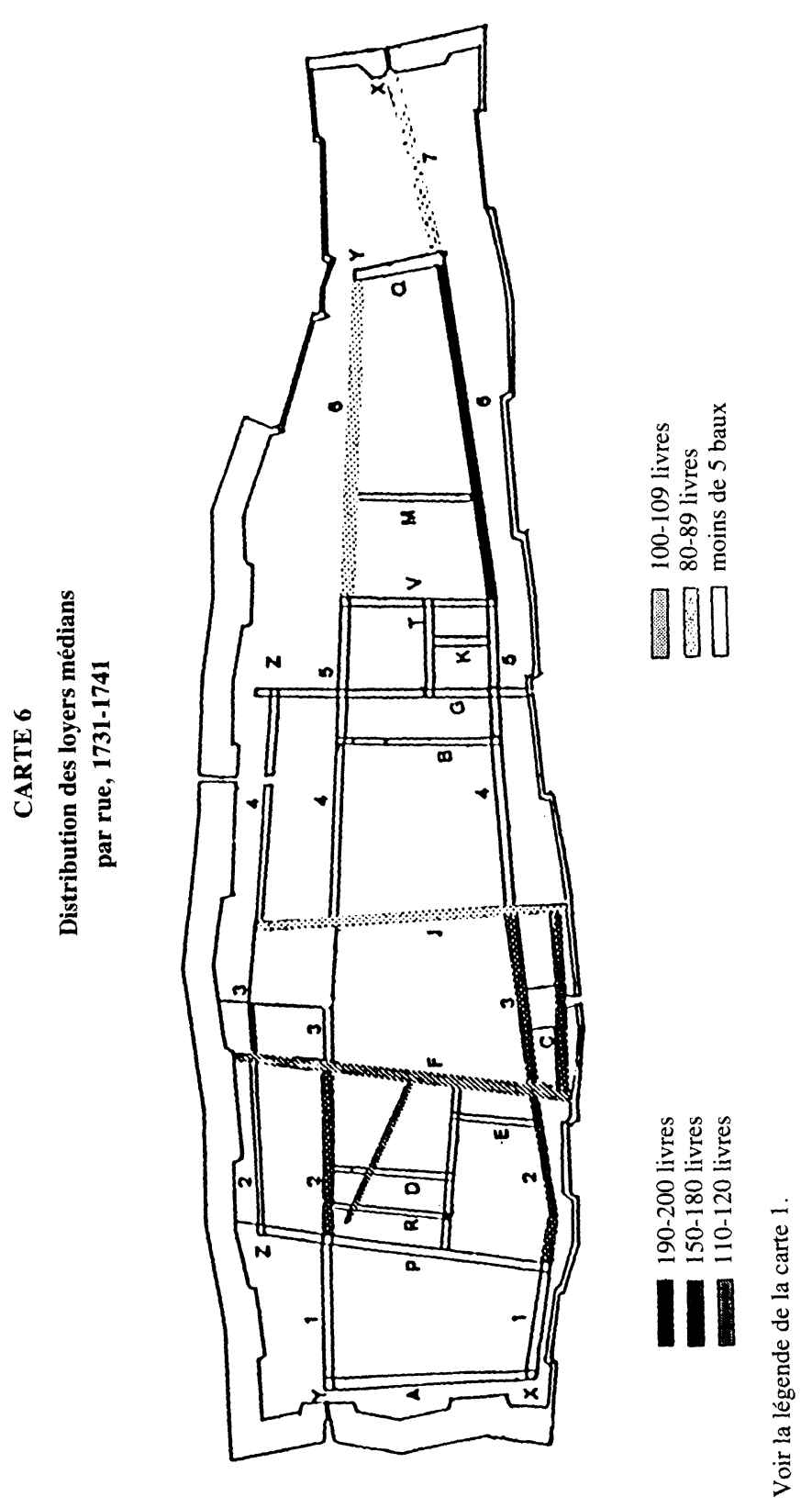


le matériau dominant, le bois, influence vraisemblablement la valeur des loyers puisque le loyer médian à 74 livres est le plus bas de la ville. Malgré le faible nombre de baux retrouvés pour ces bouts de rues (carte 2 ), nous savons que les locataires y sont nombreux en 1741 (carte 1). Comme le niveau des loyers semble correspondre à la faible capacité de payer des groupes les moins nantis de la ville, nous posons comme hypothèse que ce secteur pourrait constituer le «bas-quartier» de la ville intra-muros (que les faubourgs semblent compléter), là où résideraient les ménages ayant un niveau de vie très faible. Si notre hypothèse est fondée, nous aurons identifié un type de zone urbaine, un îlot de pauvretét ${ }^{49}$.

Le modèle concentrique que nous observons dans le tissu urbain montréalais demeure malgré tout très imparfait puisqu'il ne vaut que pour les bouts de rues occupées majoritairement par les locataires. Notre analyse doit également prendre en considération les secteurs habités majoritairement par les propriétaires résidents, par exemple le «quartier Bonsecours». Celui-ci semble évoluer en retrait du marché locatif. L'incendie de 1734 qui touche l'Hôtel-Dieu et ses abords l'a passablement séparé du secteur portuaire marchand dont il semble pourtant le prolongement naturel. Le quartier Bonsecours participe malgré tout au développement économique de la ville puisque la Compagnie des Indes y loue deux locaux aux abords de la chapelle Notre-Dame-de-Bonsecours, à raison de 600 livres chacun ${ }^{50}$. La présence de la noblesse influence probablement la forte médiane qu'atteignent les loyers de la rue St-Paul 6 (voir carte 6). En tout cas, cette petite société forme la clientèle des quelques artisans locataires retrouvés dans ce secteur, tels un perruquier et un orfèvre.

\section{CONCLUSION}

Loin de constituer un ensemble homogène de petits propriétaires, les Montréalais des années 1731 à 1741 comptent déjà au moins 35\% de ménages locataires. L'analyse de la composition socio-professionnelle des locataires et de la géographie de la location a révélé en partie la structure sociale montréalaise et ses patterns géographiques.

L'échelle des loyers a servi à identifier trois catégories socio-économiques distinctes: les groupes aisés, les gens de métier et les groupes défavorisés. Notre analyse montre que chez les plus démunis le loyer accapare plus de $40 \%$ du revenu des chefs de ménage et que, parmi eux, les femmes seules connaissent les conditions de logement les plus

49 Voir A. Sutcliffe, op.cit., 246-247. Le repérage des îlots de pauvreté des villes anglaises d'avant 1850 est d'autant plus important, selon lui, que cette condition socio-économique aurait touché environ le tiers des populations urbaines. Mais cette entreprise est particulièrement difficile dans le cas des villes pré-industrielles à cause de la rareté des sources documentaires.

50 ANQM, Gaudron de Chèvremont, 31 mai 1734; C.-J. Porlier, 30 juillet 1737. 
difficiles. Du point du vue de cette importante ponction locative, la ville industrielle ne semble donc pas avoir créé plus d'inégalités que la ville pré-industrielle. Dans l'une ou l'autre de ces deux périodes historiques, les écarts sociaux chez les locataires sont illustrés par le fait qu'un faible loyer gruge généralement une part importante du revenu des plus démunis tandis qu'un loyer coûteux ne représente qu'une petite part du revenu des plus riches.

Nous pensons avoir démontré que les images d'égalitarisme social et d'intégration spatiale résistent mal à un examen minutieux. Par la spécialisation professionnelle, par la stratification des loyers, par la concentration des locataires et celle des propriétaires résidents rencontrés dans les bouts de rues, la ville intra-muros présente un espace social différencié qui correspond à la phase d'exclusion de Vance. La division de son parc locatif en trois secteurs socio-professionnels hiérarchisés s'apparente au modèle concentrique de Sjoberg. Ainsi, nous observons une concentration de marchands dans le noyau portuaire là où les maisons de pierre et les loyers élevés prédominent, une concentration des gens de métier dans les rues adjacentes avec matériaux mixtes et loyers moyens, et une concentration des démunis dans un secteur périphérique avec construction de bois et loyers modiques qui constitue hypothétiquement un foyer d'indigence. Le tissu urbain semble donc déjà se constituer sur la base du bout de rue comme élément central de sociabilité et cela, à l'exemple de la ville industrielle ${ }^{51}$, mais à une échelle réduite.

Notre recherche ne ferme pas le dossier de la structure socio-professionnelle et de ses aspects résidentiels puisque elle ne porte que sur les locataires. Il est évident qu'une étude qui tiendrait compte de tous les résidents de telle ou telle rue serait plus révélatrice. Mais, compte tenu de l'état des sources, cette recherche du cas par cas serait difficile et laisserait sans doute des trous importants. Nous n'avons pas abordé la question de la mobilité sociale des locataires car nos données sont trop fragmentaires et portent sur une trop courte période. Néanmoins, la société entrevue dans cette étude laisse une impression de rigidité. Comme dans la ville industrielle, le passage du groupe défavorisé au groupe aisé n'est probablement l'affaire que de rares individus.

L'étude attentive de la ville pré-industrielle apparaît donc essentielle à la compréhension de la ville industrielle car ce n'est qu'en identifiant minutieusement les caractéristiques de la première que l'on pourra mesurer avec exactitude l'impact des transformations majeures qui conduisent à la seconde.

51 Voir D. Hanna et S. Olson, op. cit., 255-275. Ces auteurs utilisent une méthode semblable à la nôtre et analysent les loyers médians selon les bouts de rue. Ils montrent que les quartiers du Montréal industriel se développent non pas de façon homogène mais hétérogène, un peu comme une courte-pointe, où «l'espace de vie, c'est le bout de rue», 273. 